\title{
Development of A Self Biased High Efficiency Solid-State Neutron Detector for MPACT Applications
}

\section{Fuel Cycle R\&D}

Dr. Yaron Danon 


\section{Development of a Self-Biased High Efficiency Solid-State Neutron Detector for MPACT Applications}

FINAL REPORT August 2013

PIs: Yaron Danon, Ishwara B. Bhat, James J.-Q. Lu

Rensselaer Polytechnic Institute, Troy, NY 12180

NEUP grant number: $10-852$

Performance Period: Jun 14, 2010 to June 30, 2013

\section{Executive summary}

Neutron detection is an important aspect of Materials Protection, Accounting and Control for Transmutation (MPACT). Currently He-3 filled thermal neutron detectors are utilized in many applications; these detectors require high voltage bias for operation, which complicates the system when multiple detectors are used. In addition, due to recent increase in homeland security activity and the nuclear renaissance, there is a shortage in He-3 supply and these detectors become more expensive. Instead, cheap solid-state detectors that can be massively produced like any other computer chips were developed during this project. The new detector does not require a bias for operation, has low gamma sensitivity and fast response. The detection system is based on a honeycomb-like silicon device, which is filled with B-10 as the neutron converter; while a silicon p-n diode (i.e., solar cell type device) formed on the thin silicon wall of the honeycomb structure detects the energetic charged particles emitted from the B-10 conversion layer. The simulated detector efficiency for thermal neutrons $(0.0253 \mathrm{eV})$ of optimized structure detectors was $45 \%$. The fabricated detectors had a measured efficiency of $28 \%$ for thermal neutrons $(0.0253 \mathrm{eV})$ lower than the design simulation. The reason for this discrepancy was attributed to a non-optimal structure of the fabricated detectors. Detectors size of $8 \mathrm{~cm}^{2}$ was demonstrated using a single preamplifier and without degradation in the efficiency. This large size is essential for the construction of low-cost large area detection systems. A neutron coincidence detector was constructed using 8 detectors each with area of $4 \mathrm{~cm}^{2}$ and associated electronics. The coincidence system was tested with a Cf- 252 neutron source and compared with analytical calculations. The research project results in $2 \mathrm{PhD}$ theses, one Master's Thesis, 4 journal publications, 4 conference publications that provide detailed description of the technical work. Further the research results were presented in four professional conferences (Refs.13-17).

\section{Objectives and Accomplishments}

The project proposal had three major objectives:

1. Demonstrate a working solid-state detector with intrinsic efficiency of $\sim 40 \%$ that operates without bias voltage and has a low gamma sensitivity.

2. Demonstrate a large area detector by combining several smaller detectors that are fabricated and tested on a single wafer.

3. Demonstrate coincidence counting using the solid-state neuron detector.

A summary of the accomplishments related to each of the goals are described below: 


\subsection{Demonstrate a working solid-state detector with intrinsic efficiency of $\sim 40 \%$ that operates without bias voltage and has a low gamma sensitivity.}

This is a major goal of this project. The results are documented in several publications starting with optimization calculations [1,2]. These calculations were done in order to find the optimum geometry of such device and the highest possible efficiency. The results of this study and the good structural integrity lead to our choice of a honeycomb Si structure with holes that are about 2 um across with 2 um thick Si walls and 40 um depth. The holes in the Si were filled with B-10. References [1] and [2] also contain detailed information on the principle of operation of the detector.

The fabrication process was described in details in several publications that resulted from this research [4 - 8]. A PhD thesis provides detailed information on the earlier of fabrication process [10], another $\mathrm{PhD}$ thesis describing the current improvement to the fabrication process is still in progress.

Detectors were with surface area from 0.0625 to $16 \mathrm{~cm}^{2}$ were manufactured and tested in neutron irradiation. A neutron detection efficiency of $28 \%$ as measured with thermal neutrons $(0.0253 \mathrm{eV})$ was achieved. The nominal efficiency of most batches was about $24 \%$ and the efficiency was uniform (+/$3 \%)$ in batch production. The gamma sensitivity was measured with a Co-60 source that provided a dose of $10 \mathrm{mR} / \mathrm{h}$ and was found to be about $0.001 \%$.

\subsection{Demonstrate a large area detector by combining several smaller detectors that are fabricated and tested on a single wafer.}

The extremely low leakage current $\left[6.1 \times 10^{-9} \mathrm{Acm}^{-2}\right.$ at $\left.-1 \mathrm{~V}\right]$ of the continuous junction detector developed by our group is the key to large area detectors [5]. The current batch of detectors contained an array of $2.5 \mathrm{~mm} \times 2.5 \mathrm{~mm}$ detectors and we connected 15 detectors in parallel to from a $1 \mathrm{~cm}^{2}$ detector. We then connected in series up to 16 modules to form a $16 \mathrm{~cm}^{2}$ detector. Such detector was operated using one standard ORTEC 142AH preamplifier. The ability to construct a large area detector that requires simple electronics and no bias makes this design very attractive for large area applications. The performance degradation due to the large surface area was minimal; giving $19 \%$ efficiency with 8

$\mathrm{cm}^{2}$. This can be improved when the efficiency per device is improved with a better optimized and controlled fabrication process.

\subsection{Demonstrate coincidence counting using the solid-state neuron detector}

A neutron coincidence counter using solid-state neutron detectors can replace He-3 tubes in a conventional moderated coincidence counter [11]. For such detector to operate effectively the overall fast neutron detection efficiency is typically about $20 \%$ [11]. We constructed a prototype coincidence counter using only 8 modules of $4 \mathrm{~cm}^{2}$ detectors embedded in a cylindrical polyethylene moderator as shown in Figure 1. The detectors were connected to a Mesytec MSI-8 preamplifier-amplifier and discriminator. The signals from the 8 discriminators were connected to a logical OR grate. The logical signal from the OR gate was connect to a multi-channel scales (MCS) such that the time interval between detected neutrons was recorded. Although the overall neutron detection efficiency of such system is low and many more detectors are required for efficient operation, we were able to demonstrate that such setup will work. The distribution of time interval between pulses was compared to theoretical calculation for a random source and shows very good agreement. 


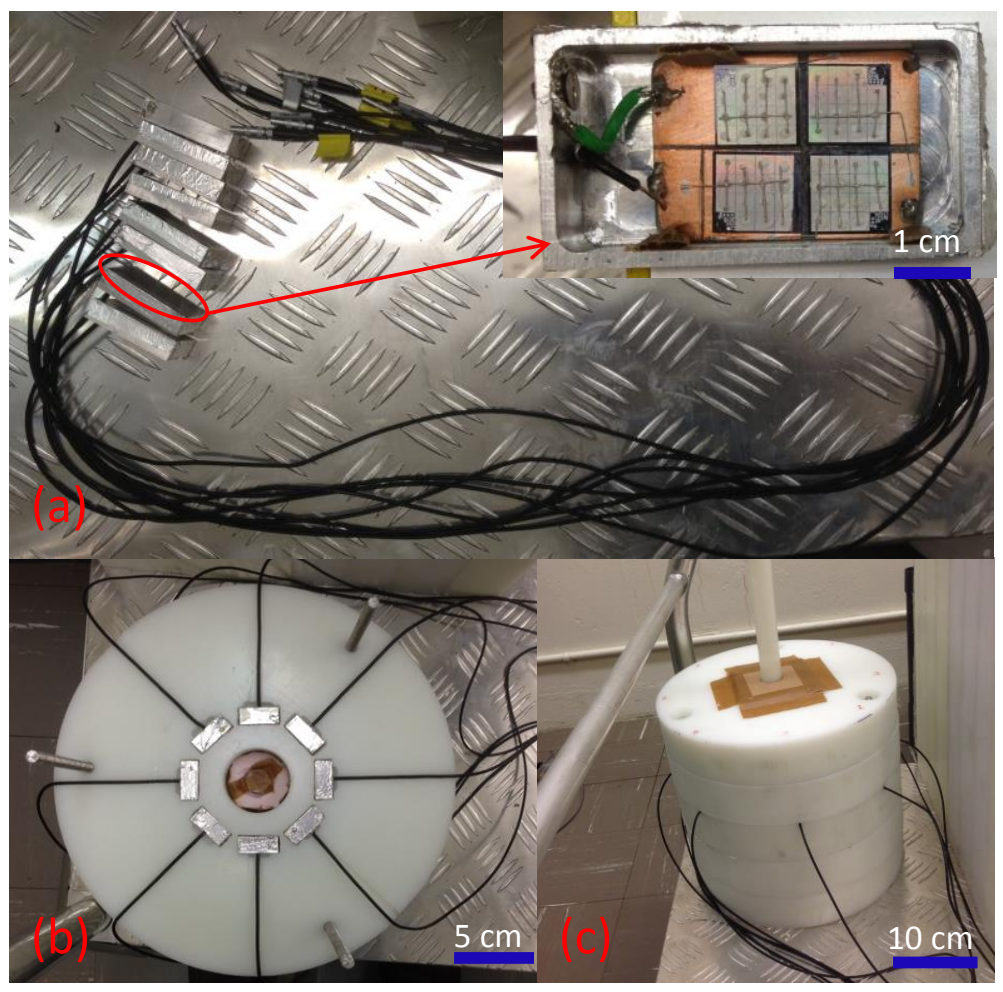

Figure 1 - (a) Eight $4 \mathrm{~cm}^{2}$ detectors module packaged in eight Al boxes with eight cables to connect to eight different channels of preamps with logical OR output. The inset shows the detector module inside an Al box, (b) Detectors embedded in HDPE around a ${ }^{252} \mathrm{Cf}$ source, and (c) the solid state coincidence counter system with ${ }^{252} \mathrm{Cf}$ source at center.

\section{References (reference not by our group are in italics)}

1. Justin Dingley, Yaron Danon, Nicholas LiCausi, Jian-Qiang Lu and Ishwara B. Bhat, "Optimization Of A Novel Solid-State Self Powered Neutron Detector", International Conference on Mathematics, Computational Methods \& Reactor Physics (M\&C 2009), Saratoga Springs, New York, May 3-7, 2009, on CD-ROM, American Nuclear Society, LaGrange Park, IL (2009).

2. Justin Clinton, "Optimization and Characterization of a Novel Self Powered Solid State Neutron Detector", PhD thesis, Rensselaer Polytechnic Institute, Troy, NY 12180, December (2011).

3. Akshay Mata, URP student, "Computer Interfacing with LabView for I-V Characterization of Boron filled Si Diodes", August 2013.

4. Kuan-Chih Huang, Rajendra Dahal, James Lu, Yaron Danon, and Ishwara Bhat, "Continuous p-n junction with extremely low leakage current for micro-structured solid-state neutron detector applications," Proceedings of SPIE 8710, Baltimore, MD, April 29-May 3 (2013).

5. Kuan-Chih Huang, Rajendra Dahal, James J.-Q. Lu, Yaron Danon, and Ishwara B. Bhat, "High detection efficiency micro-structured solid-state neutron detector with extremely low leakage current fabricated with continuous p-n junction", Appl. Phys. Lett. 102, 152107 (2013).

6. Kuan-Chih Huang, Rajendra Dahal, Nicolas LiCausi, and James J.-Q. Lu, Yaron Danon, Ishwara B. Bhat, "Boron filling of high aspect ratio holes by chemical vapor deposition for solid-state neutron detector applications", J. Vac. Sci. Technol. B 30(5), Sep/Oct (2012). 
7. R. Dahal, K. C. Huang, J. Clinton, N. LiCausi, J.-Q. Lu, Y. Danon, I. Bhat1, "Self-powered microstructured solid state neutron detector with very low leakage current and high efficiency", Applied Physics Letters 100, 243507 (2012).

8. Kuan-Chih Huang, Rajendra Dahal, James J.-Q. Lu, Yaron Danon, and Ishwara B. Bhat, "Boron Filling of Deep Holes for Solid-State Neutron Detector Applications", Transactions of the American Nuclear Society, Vol. 106, Chicago, Illinois, June 24-28, (2012).

9. Yaron Danon, Justin Clinton, Kuan-Chih Huang, Nicholas LiCausi, Rajendra Dahal, James Lu, and Ishwara Bhat, "Towards high efficiency solid-state thermal and fast neutron detectors," Journal of Instrumentation 7, C03014 (2011).

10. LiCausi, Nicholas, "Design and fabrication of a novel self-powered solid-state neutron detector", PhD thesis, Rensselaer Polytechnic Institute, Troy, NY 12180, (2011).

11. Ensslin, N., Principles of neutron coincidence counting. Passive Nondestructive Assay of Nuclear Materials, NUREG/CR-5550, (1991).

12. Jonathan Marini, "Characterization of A Novel Self-Powered Solid-State Neutron Detector" MS Thesis, Rensselaer Polytechnic Institute, Troy, NY 12180, (2011).

\section{Conference Orel Presentations}

13. Kuan-Chih Huang, Rajendra Dahal, James Lu, Yaron Danon, and Ishwara Bhat, "Continuous p-n junction With Extremely Low Leakage Current for Micro-Structured Solid-State Neutron Detector Applications," SPIE Defense, Security, and Sensing, Baltimore, MD, April 29-May 3 (2013).

14. R. Dahal, K.-C. Huang, J. Clinton, J.-Q. Lu, Y. Danon, and I. Bhat, "Advances on a high efficiency selfpowered solid state thermal neutron detection", IEEE NSS/MIC/RTSD conference, Anaheim, CA, Presentation (Oral)\#R12-6, October 29 - November 3, (2012).

15. Rajendra Dahal, Kuan-Chih Huang, James Lu, Yaron Danon, and Ishwara Bhat, "Large-area HighEfficiency Self-Powered Solid State Neutron Detector," SPIE Defense, Security, and Sensing, Baltimore, MD, April 29-May 3 (2013).

16. Kuan-Chih Huang, Rajendra Dahal, James Lu, Yaron Danon, and Ishwara Bhat, "Boron Filling of Deep Holes for Solid-State Neutron Detector Applications," American Nuclear Society Annual Meeting, Chicago, Illinois, June 24-28 (2012).

17. Ishwara Bhat, Rajendra Dahal, Kuan-Chih Huang, James Lu and Yaron Danon, "Design and Fabrication of High Efficiency Solid State Neutron Detectors", 22nd Annual Connecticut Microelectronics and Optoelectronics Consortium (CMOC) Symposium, March 13th, 2013, Yale University, New Haven, CT. (Invited). 